\title{
Contribution of the PhoP/Q regulon to survival and replication of Salmonella enterica serovar Typhimurium in macrophages
}

Correspondence

David W. Holden

d.holden@imperial.ac.uk

Sophie Helaine

s.helaine@imperial.ac.uk

Received 8 February 2011

Revised 18 April 2011

Accepted 20 April 2011

\author{
Jessica A. Thompson, Mei Liu, Sophie Helainet and David W. Holdent
}

Section of Microbiology, Centre for Molecular Microbiology and Infection, Imperial College London, Armstrong Road, London SW7 2AZ, UK

\begin{abstract}
The ability of serovars of Salmonella enterica to cause systemic disease is dependent upon their survival and replication within macrophages. To do this, bacteria must withstand or surmount bacteriostatic and bactericidal responses by the host cell, including the delivery of hydrolytic enzymes from lysosomes to the phagosome. The bacterial two-component regulatory system PhoP/Q has been implicated in avoidance of phagolysosomal fusion by $S$. enterica serovar Typhimurium (S. Typhimurium) in murine macrophages. In this study, the involvement of PhoP/Qactivated genes in avoidance of phagolysosomal fusion was analysed: of all the $S$. Typhimurium mutant strains tested, only an $\mathrm{mgtC}$ mutant strain partially reproduced the phenotype of the phoP mutant strain. As this gene is required for bacterial growth in magnesium-depleted conditions in vitro, the contributions of $\mathrm{PhoP} / \mathrm{Q}$ to intramacrophage replication and survival were reappraised. Although PhoP/Q was required for both replication and survival of $S$. Typhimurium within murine macrophages, subsequent analysis of the kinetics of phagolysosomal fusion, taking account of differences in the replication rates of wild-type and phoP mutant strains, provided no evidence for a PhoP/Q-dependent role in this process. PhoP/Q appeared to act subsequent to the process of phagolysosomal avoidance and to promote replication of those bacteria that had already escaped a phagolysosomal fate. Therefore, we conclude that the PhoP/Q regulon enables S. Typhimurium to adapt to intramacrophage stresses other than phagolysosomal fusion.
\end{abstract}

\section{INTRODUCTION}

Salmonella enterica serovar Typhi ( $S$. Typhi) causes typhoid fever, and is restricted to infection of primates. By contrast, S. enterica serovar Typhimurium (S. Typhimurium) has a broad host range and causes both self-limiting gastroenteritis and systemic diseases, depending upon the host; the systemic disease that it causes in susceptible mouse strains is frequently used as a model system to study typhoid fever. The ability of both serovars to cause systemic disease depends on their capacity to survive and grow within cells of the granulocyte/monocyte lineage, such as macrophages. Accordingly, $S$. Typhi is adapted to grow in human macrophages, while $S$. Typhimurium grows

†These authors contributed equally to this work.

Abbreviations: AMP, antimicrobial peptide; BMM, bone marrow-derived macrophages; CtsD, cathepsin D; M6PR, mannose-6-phosphate receptor; PFA, paraformaldehyde; SCV, Salmonella-containing vacuole; SPI-2, Salmonella pathogenicity island-2; TROva, Texas red ovalbumin; T3SS, type III secretion system.

Three supplementary tables, showing strains and plasmids used in this study, primers used for deletion mutagenesis, and primers used for verification of deletion and construction of complementing plasmids, are available with the online version of this paper. preferentially in mouse macrophages (Schwan et al., 2000). Two multi-functional virulence systems contribute to the intramacrophage growth and virulence of $S$. enterica: the PhoP/Q two-component system, which activates the expression of many genes following bacterial uptake into phagosomes (Groisman et al., 1989; Miller et al., 1989), and the SPI-2 type III secretion system (T3SS) (Hensel et al., 1995; Ochman \& Groisman, 1996; Shea et al., 1999), which translocates numerous effector proteins across the phagosomal membrane.

Macrophages use several bactericidal and bacteriostatic processes that must be counteracted, avoided or withstood for intracellular S. enterica to survive and replicate. DNA-, protein- and membrane-damaging reactive oxygen species are produced in the first few minutes of phagocytosis by NADPH oxidase activity. Toxic reactive nitrogen species are produced later, by inducible NO synthase (iNOS). The increased growth of $S$. Typhimurium in macrophages and knockout mouse strains lacking NADPH oxidase or iNOS demonstrates that bacteria are susceptible to these responses (Mastroeni et al., 2000; Vazquez-Torres et al., $2000 \mathrm{a}, \mathrm{b})$. This sensitivity is limited by the activity of bacterial detoxifying enzymes and regulatory factors, including catalases, hydroperoxidases (Hébrard et al., 
2009), superoxide dismutases (Craig \& Slauch, 2009; De Groote et al., 1997), HmpA, NorV, NrfA (Mills et al., 2008) and $\sigma^{\mathrm{E}}$ (Testerman et al., 2002).

A major process by which macrophages kill phagocytosed bacteria is the fusion of lysosomes to phagosomes to form phagolysosomes, acidic compartments containing proteases, glycosidases and lipases (Garin et al., 2001). Numerous studies have shown that $S$. Typhimurium modifies its phagosome, known as the Salmonella-containing vacuole (SCV), such that it acquires some features of late endosomes but remains distinct from normal phagolysosomes. Interactions with early endosomes, characterized by recruitment of early endosomal markers (Hashim et al., 2000), and acidification of the SCV (Drecktrah et al., 2006; Rathman et al., 1996, 1997) are followed by the acquisition of late endosome-associated proteins, including lysosomal membrane glycoproteins such as LAMP-1 (Becken et al., 2010; Rathman et al., 1997). However, SCVs are relatively deficient in mannose-6-phosphate receptors (M6PRs), which deliver hydrolytic enzymes to late endosomal/lysosomal compartments, and their cargo, such as cathepsin L, is also largely absent (Hashim et al., 2000; Rathman et al., 1997). Mature SCVs have also been found to be relatively inaccessible to lysosomes, as judged by co-localization with endocytotic lysosomal content using electron (Buchmeier \& Heffron, 1991) and light (Becken et al., 2010; Garvis et al., 2001) microscopy. These observations were recently confirmed using an in vitro system that analysed fusion events between purified early or mature SCVs and lysosomes. In both cases, the majority of SCVs containing viable bacteria avoided fusion (Becken et al., 2010).

Although the mechanism for avoidance of phagolysosomal fusion by $S$. Typhimurium in macrophages is not well characterized, both the SPI-2 T3SS (Uchiya et al., 1999) and the PhoP/Q regulon (Garvis et al., 2001) have been implicated. Our laboratory has reported that by $16 \mathrm{~h}$ after bacterial uptake by macrophages, an increased percentage of phoP mutant bacteria co-localizes with lysosomal markers compared with wild-type bacteria (Garvis et al., 2001). In this study we analysed genes of the PhoP/Q regulon with respect to avoidance of phagolysosomal fusion by intramacrophage $S$. Typhimurium, and reexamined the method used to measure this process. A reappraisal of the role of PhoP/Q in bacterial replication and survival leads us to conclude that the PhoP/Q regulon is not directly involved in the avoidance of phagolysosomal fusion, but is required to promote the intramacrophage replication of $S$. Typhimurium.

\section{METHODS}

Bacterial strains and growth conditions. The bacterial strains used in this study are listed in Supplementary Table S1. Bacteria were routinely grown in Luria-Bertani (LB) broth (Sambrook \& Russell, 2001) at $37^{\circ} \mathrm{C}, 200$ r.p.m., except where otherwise indicated. Antibiotics were used at the following concentrations: kanamycin,
$25 \mu \mathrm{g} \cdot \mathrm{ml}^{-1}$; carbenicillin, $50 \mu \mathrm{g} \cdot \mathrm{ml}^{-1}$; chloramphenicol, $50 \mu \mathrm{g} \cdot \mathrm{ml}^{-1}$. Protein expression was induced with $0.2 \%(\mathrm{w} / \mathrm{v})$ L-arabinose, as required (Loessner et al., 2007).

Bacterial mutagenesis. $S$. Typhimurium LT2 mutant strains were constructed using the one-step $\lambda$ red recombinase chromosomal inactivation method (Datsenko \& Wanner, 2000). Primer sequences used for targeted mutagenesis and verification of recombination are listed in Supplementary Tables S2 and S3, respectively. Clean deletions of phoP and pmrA in S. Typhimurium LT2 were generated using FLP recombinase expressed from pCP20 to excise antibioticresistance cassettes.

Plasmids. Plasmids used in this study are listed in Supplementary Table S1. pKD3 and pKD4 from Escherichia coli BW25141 (Datsenko \& Wanner, 2000) were used as PCR templates for bacterial mutagenesis. pKD46 from BW25113, and pCP20 from BT340 (Datsenko \& Wanner, 2000) were used to electroporate S. Typhimurium LT2 strains for the generation of mutant strains and the removal of antibiotic-resistance cassettes, respectively (Datsenko \& Wanner, 2000). pDiGc (Helaine et al., 2010) was electroporated into S. Typhimurium 12023 wild-type and $\Delta p h o P:$ :kan strains (Helaine et al., 2010). The vector pDSRED.T3_S4T (Sörensen et al., 2003) was used to construct pBADmgtC and pBADphoP: $m g t C$ and phoP were amplified from the $S$. Typhimurium genome with primers mgtCNdeI-F and mgtCHindIII-R, or phoPXbaI-F and phoPHindIIIR (Supplementary Table S2), which introduced a NdeI, HindIII or $X b a \mathrm{I}$ restriction site flanking the genes. Vector and PCR products were restriction-digested, and inserts were ligated into the vector in place of the excised dsred.T3_S4T gene under the regulation of the arabinose-inducible $\mathrm{P}_{\mathrm{BAD}}$ promoter.

Identification of lysosomal compartments. RAW264.7 (91962702) macrophage-like cells purchased from the European Collection of Cell Cultures were seeded on glass coverslips in 24-well plates at a density of $1 \times 10^{5}$ cells per well. Cells were incubated for 12-24 h in Dulbecco's modified Eagle's medium (DMEM) supplemented with $10 \%(\mathrm{v} / \mathrm{v})$ fetal calf serum (FCS) at $37{ }^{\circ} \mathrm{C}, 5 \% \mathrm{CO}_{2}$, prior to experiments. To label lysosomes, macrophages were pulsed for $30 \mathrm{~min}$ with DMEM/10\% FCS containing $50 \mu \mathrm{g} \mathrm{ml}^{-1}$ Texas red ovalbumin (TROva; Molecular Probes, Invitrogen), washed, and incubated for $2 \mathrm{~h}$ in label-free DMEM/10 \% FCS (Garvis et al., 2001) before fixation with $3 \%(\mathrm{w} / \mathrm{v})$ paraformaldehyde (PFA) for $15 \mathrm{~min}$ at $25{ }^{\circ} \mathrm{C}$. Cells were permeabilized with $0.1 \%(\mathrm{w} / \mathrm{v})$ saponin in PBS and labelled with a $1: 500$ dilution of rabbit anti-cathepsin D (anti-CtsD) primary antibody (provided by S. Kornfeld, Washington University) and a 1:200 dilution of anti-rabbit Cy2-conjugated secondary antibody (all conjugated secondary antibodies were purchased from Jackson Immunoresearch Laboratories). Co-localization between CtsD and TROva was analysed in a minimum of 50 cells per coverslip by laser-scanning confocal microscopy (Zeiss Axiovert LSM510).

Assay for SCV-lysosome interactions in macrophages. RAW264.7 macrophages were seeded, and lysosomes were labelled with TROva as described above. Cells were infected at an m.o.i. of $10: 1$ with bacteria grown to stationary phase and opsonized as previously described (Beuzón et al., 2000). A positive control containing heat-killed bacteria $(500 \mu$ l stationary phase wild-type $S$. Typhimurium culture, incubated at $65{ }^{\circ} \mathrm{C}$ for $25 \mathrm{~min}$ ) was similarly opsonized and used for infection. The lack of viability of these bacteria was confirmed by an absence of detectable c.f.u. on LB agar (Sambrook \& Russell, 2001). At the time points indicated, infected macrophages were fixed with PFA, permeabilized with $0.1 \%$ saponin in PBS, and labelled with a $1: 200$ dilution of goat anti-Salmonella CSA-1 primary (Kirkegaard and Perry Laboratories) and $1: 200$ antigoat Cy2-conjugated secondary antibodies. Co-localization of heat- 
killed bacteria and CtsD was detected in macrophages labelled with anti-CtsD antibody and a 1:400 dilution of anti-rabbit Rhodamine Red-X (RRX)-conjugated secondary antibodies. Pixel-to-pixel colocalization between bacteria and lysosomal markers was assessed by laser-scanning confocal microscopy in a minimum of 50 cells per coverslip. Macrophages containing in excess of approximately 25 bacteria were excluded from the analysis due to the difficulty in accurately determining their number and the proportion co-localizing with TROva.

Bacterial growth in RAW264.7 macrophages. RAW264.7 macrophages were infected for different time periods with opsonized stationary-phase cultures of bacteria at an m.o.i. of $10: 1$, as described above. Cells were lysed with $0.1 \%(\mathrm{v} / \mathrm{v})$ Triton X-100 in PBS, and c.f.u. were enumerated by plating serial dilutions of the lysate on LB agar. Bacterial growth was measured as the fold change in c.f.u. $\mathrm{ml}^{-1}$ recovered from macrophages between two time points.

Bacterial replication and survival in macrophages. Bone marrow-derived macrophages (BMM) were extracted from BALB/c mice (Charles River) and cultured as described by Helaine et al. (2010). BMM medium [RPMI 1640 (Invitrogen) supplemented with $10 \%$ (v/v) FCS Gold (PAA laboratories), $2 \mathrm{mM}$ glutamine, $50 \mu \mathrm{M} \beta$ mercaptoethanol, $1 \mathrm{mM}$ sodium pyruvate and $10 \mathrm{mM}$ HEPES] was used to maintain BMM, which were seeded at a density of $2 \times 10^{5}$ cells per well in 24-well plates. RAW264.7 macrophages or BMM were infected with bacterial strains carrying the pDiGc plasmid grown to late stationary phase in MgM-MES pH 5.0 [170 mM MES, pH 5.0, $5 \mathrm{mM} \mathrm{KCl}, 7.5 \mathrm{mM}\left(\mathrm{NH}_{4}\right)_{2} \mathrm{SO}_{4}, 0.5 \mathrm{mM} \mathrm{K}_{2} \mathrm{SO}_{4}, 1 \mathrm{mM} \mathrm{KH_{2 }} \mathrm{PO}_{4}$, $10 \mathrm{mM} \mathrm{MgCl}_{2}, 38 \mathrm{mM}$ glycerol and $0.1 \%$ Casamino acids (Beuzón et al., 1999)], in the presence of $0.2 \% \mathrm{~L}$-arabinose and antibiotics as required. At specified time points, cells were washed and lysed, and bacterial growth was quantified by enumeration of c.f.u. $\mathrm{ml}^{-1}$. The remaining lysate was centrifuged at $10000 \mathrm{~g}$, bacteria were resuspended in $500 \mu \mathrm{l} \mathrm{PBS}$, and the levels of DsRED and GFP fluorescence intensity were analysed by flow cytometry. Replication was measured as the fold change in the geometric mean of DsRED fluorescence intensity between two time points. Killing indices were calculated as the difference between replication rate and net growth rate in number of generations per hour, using the relationship $F=2^{n}$, where $n$ corresponds to the number of generations and $F$ is the fold change between the two time points.

Flow cytometric acquisition and analysis. Bacterial samples were analysed using a FACSCalibur (Beckton Dickinson) for fluorescence intensities in the FL-1 (GFP) and FL-2 (DsRED) channels; a minimum of 10000 bacterial events were analysed for each sample. Data were analysed using FlowJo 8.6.3 software. The bacterial population was selected on the basis of GFP expression; DsRED fluorescence intensity was analysed across the bacterial population, and the geometric mean of DsRED fluorescence was used to calculate the dilution of fluorescence (Helaine et al., 2010).

Statistical analysis. All data shown are either the mean and SD of a minimum of three independent experiments, or data from a single, representative experiment, reproduced on a minimum of three independent occasions. Statistical significance was calculated using Student's $t$ test, where $P<0.05$ was judged to be significant.

\section{RESULTS AND DISCUSSION}

\section{Visualization of lysosomes in macrophages}

We first compared two methods for detecting lysosomes by light microscopy. In one, a fluid-phase marker, TROva, was pulse-chased into lysosomes. Cells were then fixed and labelled for the lysosomal enzyme CtsD (Garvis et al., 2001). Analysis by confocal microscopy showed that both labels had a vesicular distribution (Fig. 1a). Almost all cells $(99 \%)$ contained at least partial pixel-to-pixel co-localization in the labelling patterns produced by TROva and CtsD (Fig. 1b). Although full co-localization was restricted to $28 \%$ of cells, $71 \%$ contained partial overlap, i.e. duallabelled TROva- and CtsD-positive compartments (Fig. 1a, white arrow), as well as compartments distinctly labelled for one or the other marker (Fig. 1a, red arrow).

TROva is taken up from the extracellular environment and traffics through early and late endosomes before reaching lysosomes, whereas CtsD is delivered by the M6PRs to late endosomes from the trans-Golgi network (Ghosh et al., 2003). Acidification triggers the release of the pro-enzyme from the receptor, whereupon cleavage generates the mature, hydrolytic protein found in the lysosomal lumen. One explanation for the lack of complete co-localization between these two markers is the ability of the anti-CtsD antibody to bind the immature, pro-form of the enzyme (data not shown). This would lead to the identification of pre-lysosomal compartments in addition to lysosomes containing the mature version of CtsD. Furthermore, lysosomes undergo fusion and kiss and run events with autophagosomes (Jahreiss et al., 2008) and late endosomes (Mullock et al., 1998, 2000; Pryor et al., 2000). Differences in the sorting of TROva and CtsD at this stage might also give rise to the distinct patterns of labelling observed.

We next determined the extent of co-localization of these markers with positive and negative controls for avoidance of phagolysosomal fusion: viable or heat-killed $S$. Typhimurium strain LT2 in RAW264.7 macrophages fixed $2 \mathrm{~h}$ after uptake. The majority of viable bacteria avoided co-localization with either marker, with $28 \%$ positive for CtsD compared with the $16 \%$ positive for TROva (Fig. 1c). In contrast, $59 \%$ and $82 \%$ of heat-killed bacteria were positive for CtsD labelling and TROva, respectively (Fig. 1c). In view of the better discrimination between negative and positive controls obtained with TROva, this probe was used in subsequent experiments to assess levels of fusion between SCVs and lysosomes.

Due to the availability of the entire genome sequence of the S. Typhimurium LT2 strain at the time this study was initiated, it was decided to use this strain to construct the pag mutant strains. To validate the use of the LT2 strain, we first constructed a phoP mutant in this strain. The phenotype of the LT2 phoP mutant was indistinguishable from that of the 12023 phoP mutant used previously (Garvis et al., 2001) with respect to intramacrophage growth and co-localization with TROva (Fig. 2a, b). Growth of both 12023 and the LT2 phoP mutant strain was restored, and the percentage of mutant bacteria that co-localized with TROva reduced from approximately 40 to $20 \%$, by the expression of phoP from pBADphoP (Fig. $2 \mathrm{a}, \mathrm{b})$, showing plasmid-based complementation. 
(a)
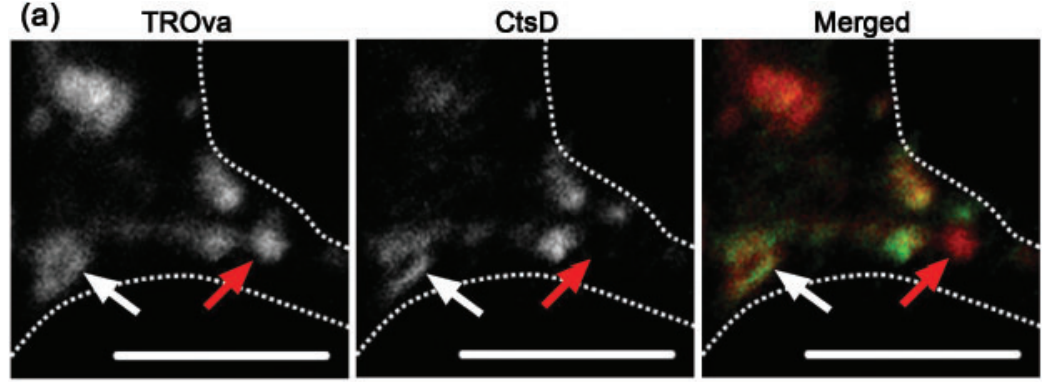

(b)

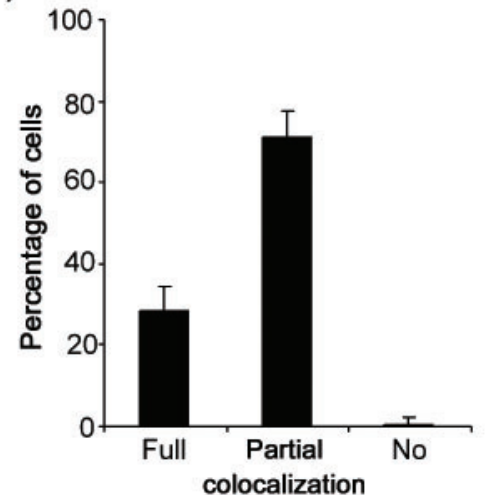

(c)

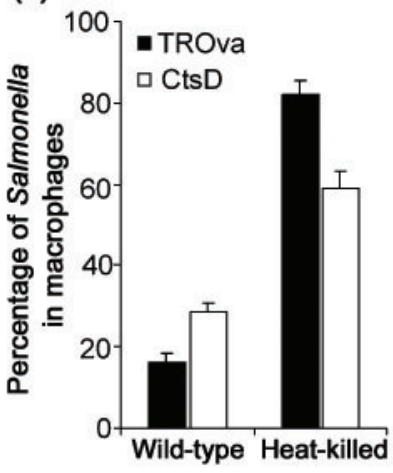

Fig. 1. Identification of lysosomes in RAW264.7 macrophages. (a) Confocal microscopy images of a RAW264.7 macrophage pulse-chased with TROva (red in merged image) and labelled with anti-CtsD antibodies (green in merged image). The dotted white line indicates the cell membrane, white arrows indicate co-localization and red arrows indicate the absence of co-localization; bars, $5 \mu \mathrm{m}$. (b) Percentage of RAW264.7 macrophages containing full, partial or no co-localization between TROva and CtsD labelling. Data shown are the mean of three independent experiments in which a minimum of 50 cells were scored; error bars, SD. (c) Percentage of viable and heat-killed S. Typhimurium LT2 colocalized with TROva or anti-CtsD labelling in RAW264.7 macrophages $2 \mathrm{~h}$ after uptake. Data shown are the mean of at least three independent experiments; error bars, SD.

\section{A pag mutant strain partially reproduces the phenotype of the phoP mutant strain}

Using the LT2 strain, mutations were made in pmrA, genes involved in modification of LPS, magnesium homeostasis, antimicrobial peptide (AMP) resistance, and genes with less well-characterized functions. TROva-labelled RAW264.7 macrophages were infected with wild-type or mutant bacteria for $18 \mathrm{~h}$, or heat-killed bacteria for $2 \mathrm{~h}$. Characteristic images of viable bacteria avoiding phagolysosomal fusion, and a non-viable bacterium exhibiting full colocalization, are shown in Fig. 3(a). For almost all the mutant strains analysed, the percentage of bacteria colocalizing with TROva was similar to that obtained for wildtype bacteria (Fig. 3b). The pagO mutant strain had a significantly lower percentage of co-localization compared with the wild-type strain. However, since the purpose of the screen was to identify mutants that could account for the phoP mutant phenotype, this was not studied further. Only the $m g t C$ mutant strain exhibited a significantly higher mean percentage of co-localization: $38 \%$ of bacteria per cell were positive for phagolysosomal fusion, compared with $50 \%$ for the phoP mutant and $15 \%$ for the wild-type strain. This phenotype was rescued by the expression of $m g t C$ from pBADmgtC (Fig. 3b).

$m g t C$ encodes a $23 \mathrm{kDa}$ protein required for $S$. Typhimurium growth within macrophages (Blanc-Potard \& Groisman, 1997; Rang et al., 2007). In addition, an $m g t C$ mutant strain has also been found to be deficient for growth in magnesium-deprived conditions in vitro (BlancPotard \& Groisman, 1997). $m g t C$ is in an operon that includes the magnesium transporter-encoding gene $m g t B$
(Hmiel et al., 1989), and although there is no evidence that supports a direct role for $\mathrm{MgtC}$ as an ion transporter (Günzel et al., 2006; Moncrief \& Maguire, 1998), the putative inner membrane location of MgtC (Rang et al., 2007), the importance of this protein for in vitro growth, and the partial rescue of intramacrophage growth of $m g t C$ mutant bacteria upon addition of $\mathrm{MgCl}_{2}$ (Blanc-Potard \& Groisman, 1997), all suggest that MgtC promotes growth within environments limited in metal ions. It is therefore unclear how it might enable avoidance of phagolysosomal fusion. In view of this result, we reappraised the apparent contribution of PhoP/Q to survival and replication in macrophages (Garvis et al., 2001).

\section{Contribution of PhoP to replication in macrophages}

As LT2 and 12023 S. Typhimurium strains displayed similar phenotypes in terms of lysosomal co-localization and propagation in macrophages (Fig. 2), and since the kinetics of replication, killing and overall growth of the 12023 strain had already been thoroughly characterized in macrophages (Helaine et al., 2010), we assessed the contribution of PhoP/Q to replication using strain 12023. RAW264.7 macrophages were infected with wild-type or phoP mutant strains carrying pDiGc. This plasmid encodes constitutive GFP as a marker for bacterial cells, and arabinose-inducible DsRED, the preformed pool of which is diluted between daughter cells with each bacterial division upon removal of the inducer. Replication is quantified according to decreases in DsRED fluorescence intensity (Helaine et al., 2010). Dilution of DsRED 

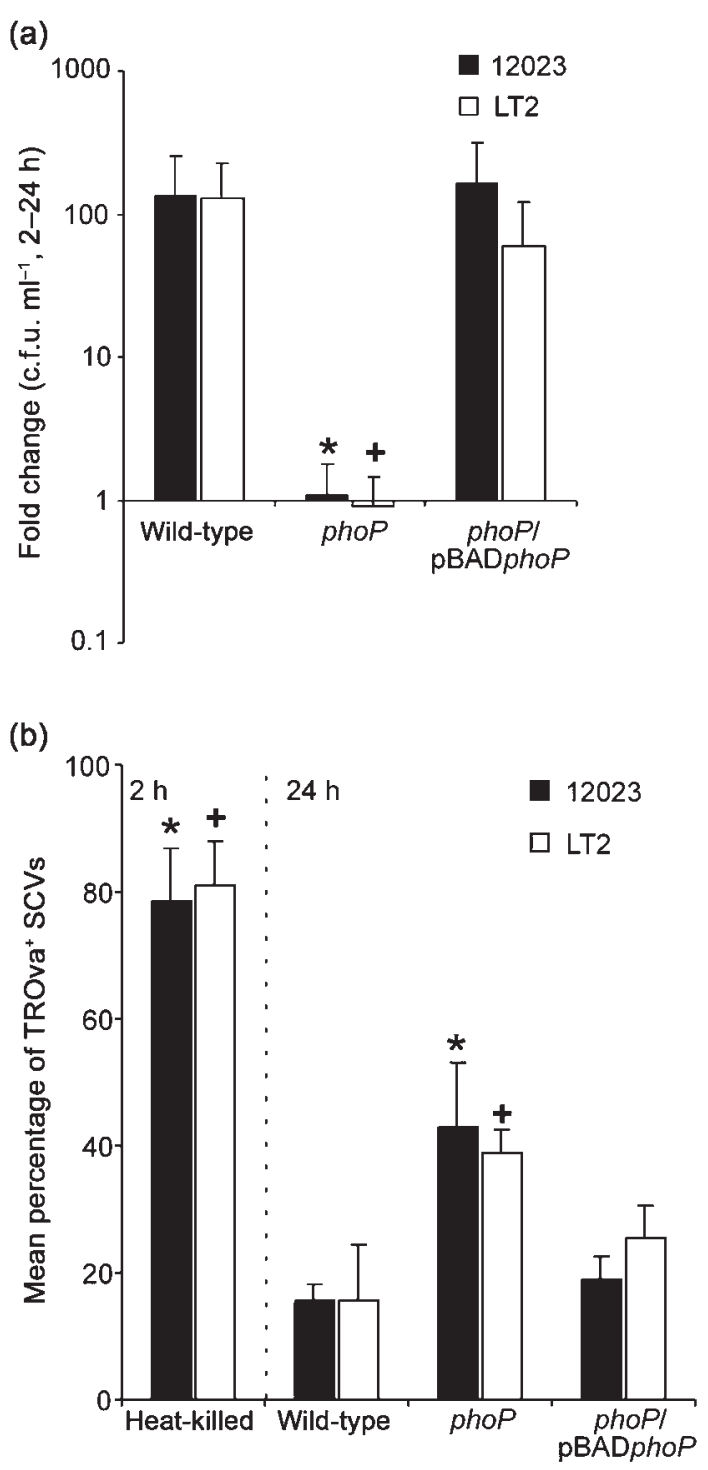

Fig. 2. S. Typhimurium LT2 phoP mutant phenotype. (a) RAW264.7 macrophages were infected with 12023 or LT2 wildtype, phoP or phoP/pBADphoP mutant $S$. Typhimurium in the presence of $0.2 \%$ L-arabinose. Net growth between 2 and $24 \mathrm{~h}$ was calculated from the fold change in c.f.u. $\mathrm{ml}^{-1}$ recovered at these time points. (b) TROva-labelled RAW264.7 macrophages were infected with wild-type, phoP or phoP/pBADphoP mutant $S$. Typhimurium in the presence of $0.2 \%$ L-arabinose for $24 \mathrm{~h}$. A positive control containing heat-killed wild-type $S$. Typhimurium was analysed $2 \mathrm{~h}$ post-uptake. The mean percentage of bacteria (as identified by antibody labelling) per cell that co-localized with TROva was scored by confocal microscopy. Data shown in (a) and (b) are the mean of three independent experiments (error bars, SD), and significant differences from the wild-type are indicated by an asterisk (12023) or cross (LT2), where $P<0.05$.

fluorescence in $S$. Typhimurium recovered from RAW264.7 macrophages was monitored by flow cytometry over a $16 \mathrm{~h}$ period and bacterial replication calculated from the fold change in geometric mean fluorescence. This revealed an approximately 100-fold increase in wild-type bacteria between 2 and $16 \mathrm{~h}$ (Fig. 4a), which corresponded to a mean rate of 0.46 generations $h^{-1}$. In contrast, the phenotype of phoP mutant bacteria became apparent $4 \mathrm{~h}$ post-uptake, with the bacteria exhibiting almost no replication (Fig. 4a) and a mean of approximately one cell division over the full course of the experiment.

Single-cell analysis by flow cytometry of bacteria recovered from RAW264.7 macrophages showed that wild-type $S$. Typhimurium replicated in a relatively synchronous wave, as evidenced by the high frequency of events within a narrow range of fluorescence intensities. This is shown for bacteria recovered after $8 \mathrm{~h}$ of infection (Fig. 4b, left panel, grey profile), but was consistently reproduced at each time point analysed (data not shown and Helaine et al., 2010). In contrast, wild-type bacteria recovered from BMM after $24 \mathrm{~h}$ exhibited a broader, more heterogeneous distribution of fluorescence intensities and therefore replicative capability (Fig. 4b, left panel, black profile). Presumably this reflects the increased stress to which bacteria were subjected in BMM compared with RAW264.7 macrophages, and was accompanied by only a 10-fold increase in bacteria in these cells (data not shown and Helaine et al., 2010).

The distribution of fluorescence intensities of phoP mutant bacteria in RAW264.7 macrophages or BMM over a $24 \mathrm{~h}$ period was similar, revealing a heterogeneous distribution of fluorescence intensity, and therefore replicative ability, across the bacterial population (Fig. 4b, right panel). The extent of replication of phoP mutant bacteria in BMM macrophages was similar to that in RAW264.7 cells (data not shown). The profiles of phoP mutant bacteria in BMM and RAW264.7 macrophages (Fig. 4b, right panel) more closely resembled those of wild-type bacteria in BMM rather than RAW264.7 macrophages (Fig. 4b, left panel), indicating that the phoP mutant strain sustains a similar degree of stress, with consequent effects upon replication, irrespective of macrophage type.

We then analysed whether this effect upon the replicative potential of phoP mutant bacteria was accompanied by an increased susceptibility to killing. Differences between the rates of replication and net growth for wild-type and phoP mutant strains were used to calculate killing indices (Helaine et al., 2010). Relatively little bacterial loss was observed for wild-type $S$. Typhimurium recovered from RAW264.7 macrophages between 2 and $24 \mathrm{~h}$, generating a killing index of 0.08 generations $\mathrm{h}^{-1}$ (Fig. 4c). This almost tripled to 0.21 generations $\mathrm{h}^{-1}$ for bacteria recovered from BMM (Fig. 4c; Helaine et al., 2010), confirming that these macrophages have a greater bactericidal capacity. Despite this difference in killing activity, there was no difference in the relatively high killing indices obtained for phoP mutant bacteria in the different macrophages, representing a loss of 0.16 generations $\mathrm{h}^{-1}$ between 2 and $24 \mathrm{~h}$ (Fig. 4c). This shows that bacteria lacking $\mathrm{PhoP}$ are highly sensitive to 
(a)
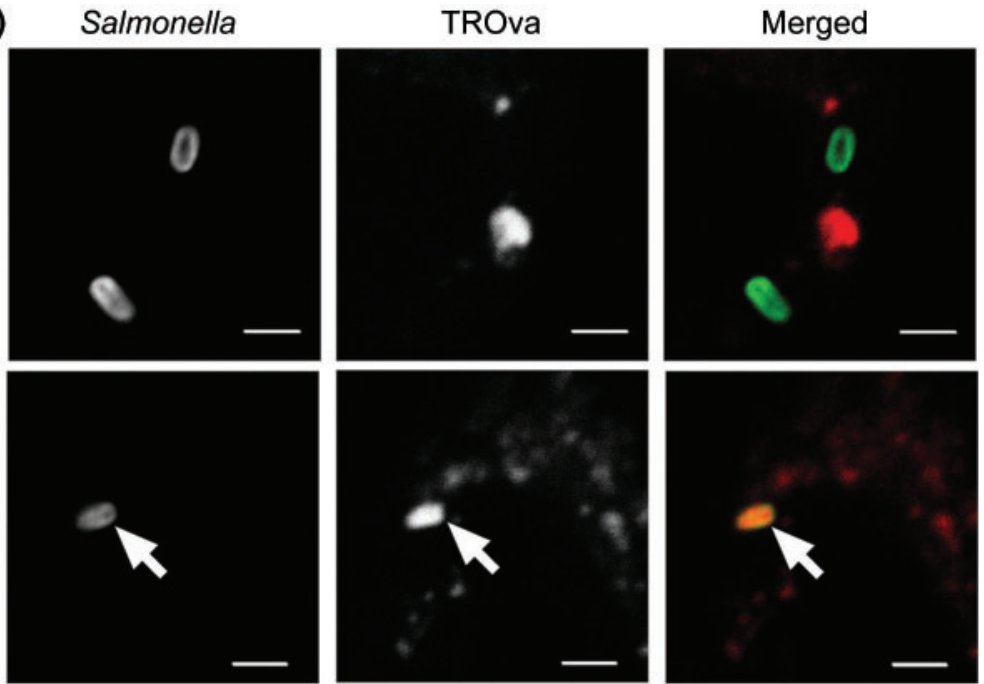

(b)

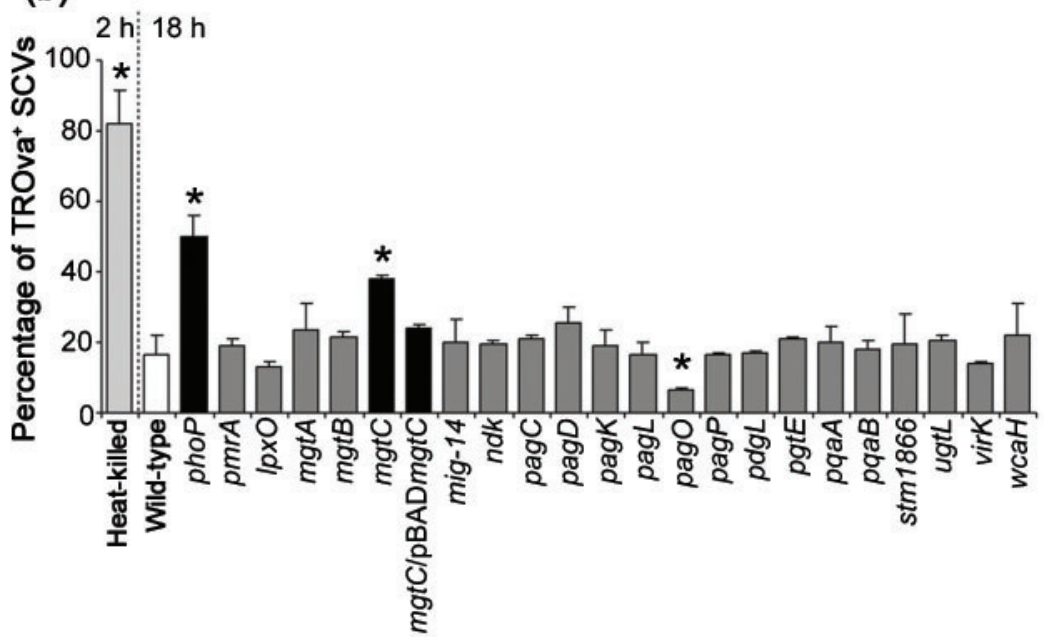

Fig. 3. Analysis of TROva co-localization with pag-mutant $S$. Typhimurium strains in macrophages. (a) Characteristic images of $S$. Typhimurium (green in merged images) negative for TROva (red in merged images) colocalization (top panel) and heat-killed $S$. Typhimurium positive for co-localization (bottom panel) in RAW264.7 macrophages. Bars, $2 \mu \mathrm{m}$; arrow indicates TROva ${ }^{+}$bacterium. (b) Mean percentage of $S$. Typhimurium per cell co-localized with TROva in RAW264.7 macrophages infected with the indicated bacterial strains for $2 \mathrm{~h}$ (heat-killed) or $18 \mathrm{~h}$ (all other strains). Data shown are the mean of at least three independent experiments (error bars, SD), and asterisks indicate a significant difference from the wild-type, where $P<0.05$. both RAW264.7 macrophages and BMM, and that the increased bactericidal capacity of BMM has no greater effect on this mutant strain. Therefore, the factors to which the PhoP/Q regulon enables intracellular adaptation are likely to be a common feature of RAW264.7 macrophages and BMM.

\section{Kinetics of avoidance of phagolysosomal fusion}

As the phoP mutant strain appeared to be equally stressed in RAW264.7 macrophages and BMM, despite the increased bactericidal activity of the latter, we re-examined the kinetics of phagolysosomal fusion in RAW264.7 macrophages at early and late time points for both wildtype and phoP mutant $S$. Typhimurium. Macrophages were pulse-chased with TROva and infected with opsonized bacteria. Between 25 and $17 \%$ of wild-type bacteria per cell co-localized with TROva at selected time points throughout an $18 \mathrm{~h}$ infection (Fig. 5a). Although the percentage of phoP mutant bacteria co-localized with TROva increased with time, reaching 45 and $48 \%$ of bacteria by 14 and $18 \mathrm{~h}$ post-inoculation, respectively, in broad agreement with previous work from our laboratory (Garvis et al., 2001), the percentage of co-localization at $2 \mathrm{~h}$ was very similar to that of the wild-type strain (Fig. 5a). In contrast, almost $70 \%$ of heat-killed bacteria were positive for TROva labelling at 2 and $6 \mathrm{~h}$ post-inoculation, indicating rapid phagolysosome maturation.

To take account of the observed difference in the replication rates of the wild-type and phoP mutant strains (Fig. 4a) in the analysis of phagolysosomal fusion, the total numbers of bacteria per macrophage, and those colocalized with TROva, were compared by microscopy (Fig. 5b). A significant difference between wild-type and phoP mutant bacteria was apparent in the total number of bacteria per cell: from $6 \mathrm{~h}$ onwards an increase in the number of wild-type bacteria per cell was observed, which reached a mean bacterial load of 14-16 bacteria per cell by 14 and $18 \mathrm{~h}$ (Fig. 5b); at these time points, macrophages infected with phoP mutant $S$. Typhimurium only contained 
(a)

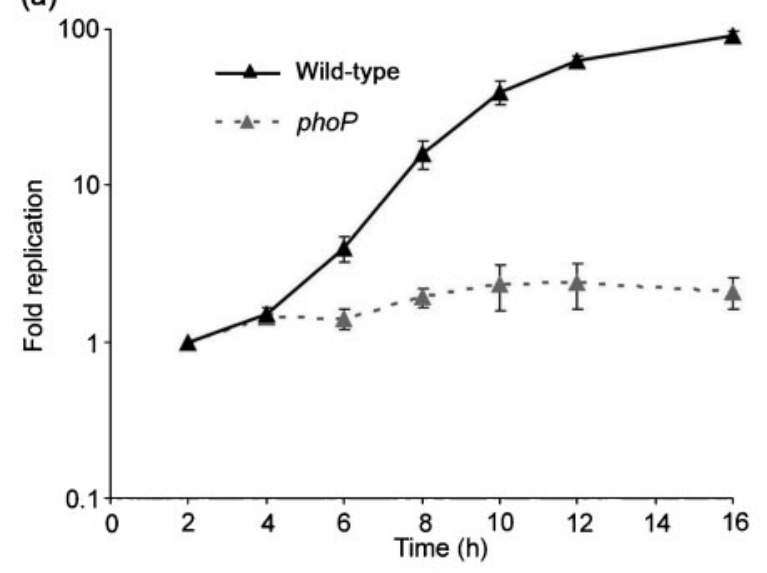

(b)
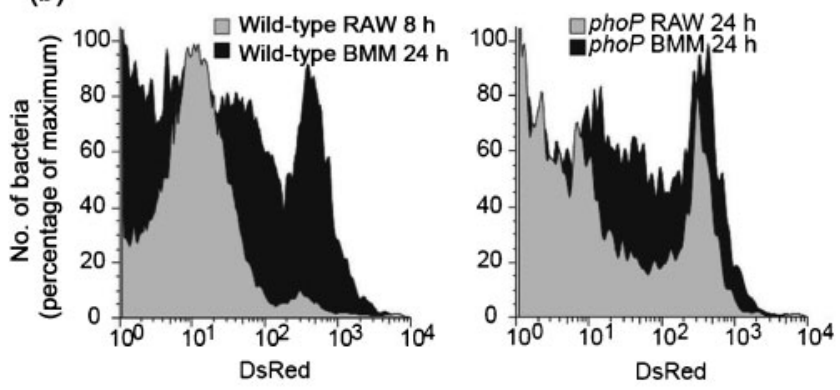

(c)

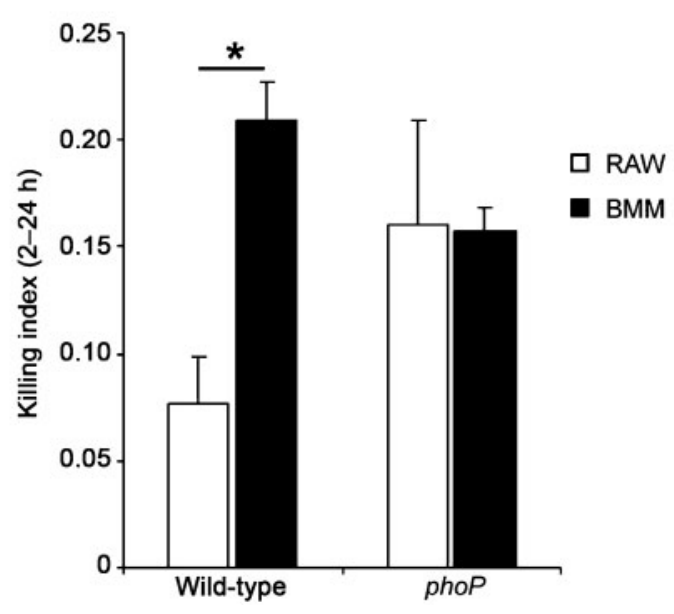

Fig. 4. $P h o P / Q$ is required for $S$. Typhimurium replication in macrophages. (a) Replication, quantified from the fold change in fluorescence, of wild-type and phoP mutant pDiGc S. Typhimurium in RAW264.7 macrophages. Data shown are the mean of three independent experiments; error bars, SD. (b) Representative histograms of the fluorescence intensity of wild-type (left) or phoP mutant (right) pDiGc $S$. Typhimurium recovered from RAW264.7 macrophages and BMM at 8 and $24 \mathrm{~h}$, as indicated. (c) Killing indices (generations $\mathrm{h}^{-1}$ ) of wild-type and phoP mutant pDiGc S. Typhimurium in RAW264.7 cells and BMM between 2 and $24 \mathrm{~h}$. Data shown are the mean of three independent experiments (error bars, SD), and asterisks indicate a significant difference between samples, where $P<0.05$. seven to eight bacteria. This difference in the intracellular numbers of wild-type and phoP mutant strains is also in agreement with their divergent kinetics of replication measured by fluorescence dilution (Fig. 4a).

Whether infected with wild-type or phoP mutant $S$. Typhimurium, macrophages contained a mean of four to five bacteria at $2 \mathrm{~h}$ post-uptake. In each case, one to two of these bacteria had co-localized with TROva (Fig. 5b). As the infection proceeded, the number of TROva-positive bacteria only increased by approximately one per cell, and at no point was there a significant difference in the number of wild-type or phoP mutant $S$. Typhimurium positive for TROva labelling (Fig. 5b). Therefore, we conclude that the PhoP/Q regulon does not directly affect SCV-lysosome interactions, but does enable proliferation of the nonlysosomal population of bacteria. As a result, the few phoP mutant bacteria undergoing phagolysosomal fusion constitute a greater proportion of the total intracellular population than do phagolysosomal wild-type bacteria, consistent with previous results (Garvis et al., 2001). These interpretations are summarized in Fig. 5(c), and highlight how differences in the intracellular growth of bacterial strains can generate misleading conclusions when data are expressed as a proportion of the population rather than in absolute terms.

Although there was no detectable difference between wildtype and phoP mutant bacteria, the number of heat-killed bacteria that co-localized with TROva was significantly higher than that of the wild-type at $6 \mathrm{~h}$ post-uptake. This indicates that there are factors other than the PhoP/Q regulon that enable $S$. Typhimurium to escape phagolysosomal fusion. Although the SPI-2 T3SS has been implicated in this phenomenon (Uchiya et al., 1999), a recent study by our group indicates that the SPI-2 T3SS is not involved in resistance to macrophage killing mechanisms (Helaine et al., 2010), and future work is required to establish the identity of the relevant bacterial molecules.

Genes of the PhoP/Q regulon are involved in several functions, including magnesium transport (Blanc-Potard \& Groisman, 1997; Soncini et al., 1996). AMPs, produced by a range of host cells including macrophages, have activity against S. Typhimurium (Beuzón et al., 2002; Blanc-Potard \& Groisman, 1997; Hiemstra et al., 1993, 1999; Soncini et al., 1996). In vitro studies indicate that some PhoPactivated genes (pags), including mig-14, virK and pagP, increase resistance to AMPs such as polymyxin B and protegrin-1 (Brodsky et al., 2002; Detweiler et al., 2003; Guo et al., 1998); the partial rescue of growth of a phoP mutant strain in BMM lacking the AMP CRAMP suggests that this also occurs in the intramacrophage environment (Rosenberger et al., 2004). Components of the PhoP/Q regulon have been shown to be involved in the tolerance response to inorganic acid stress at $\mathrm{pH} 4.5$ (Bearson et al., 1998). The $\mathrm{pH}$ of the SCV drops rapidly to between $\mathrm{pH} 4$ and 5 (Rathman et al., 1996). Therefore, an acid tolerance response is likely to be important for $S$. Typhimurium to 


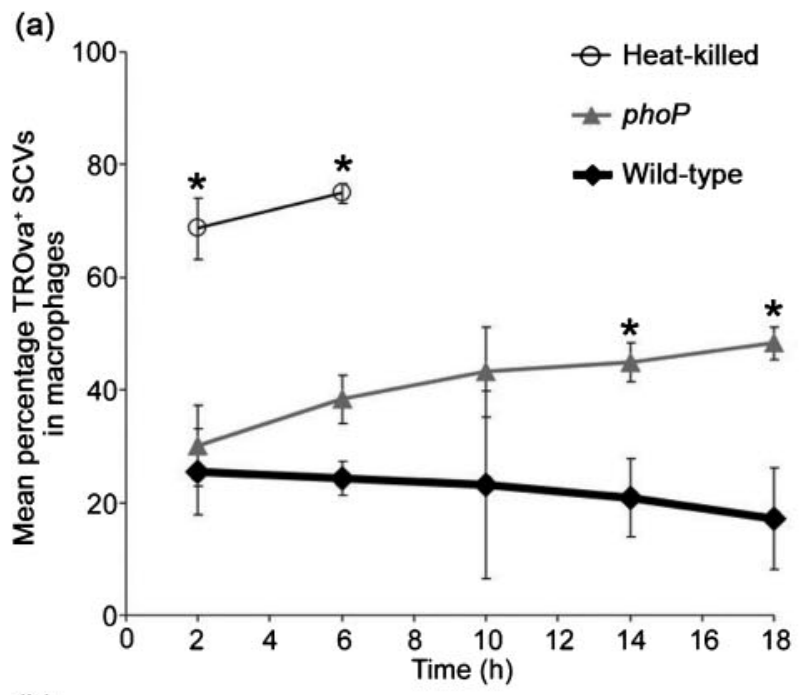

(b)

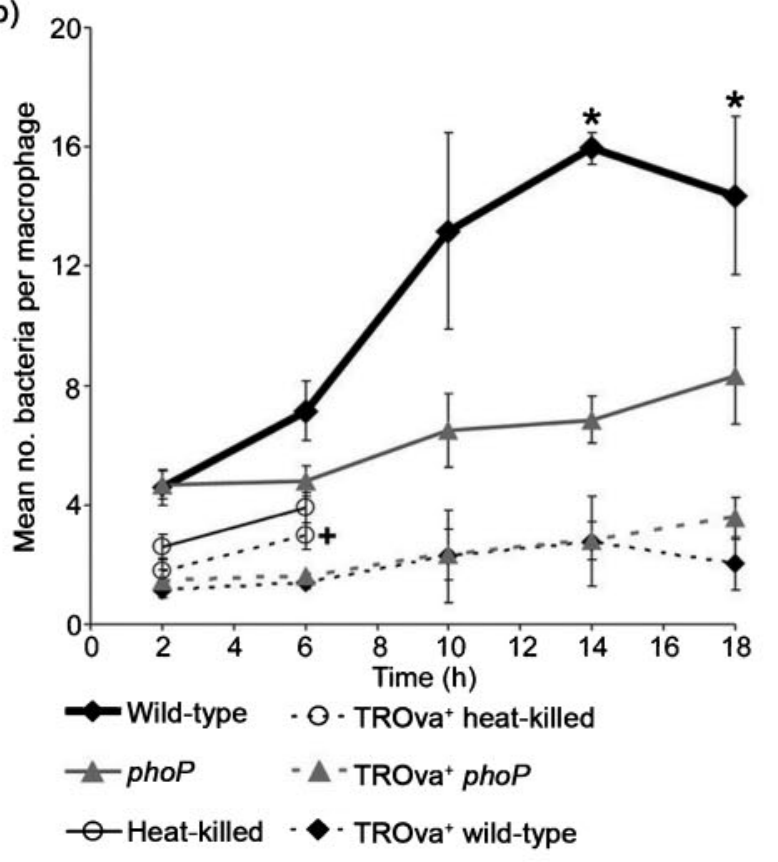

(c)
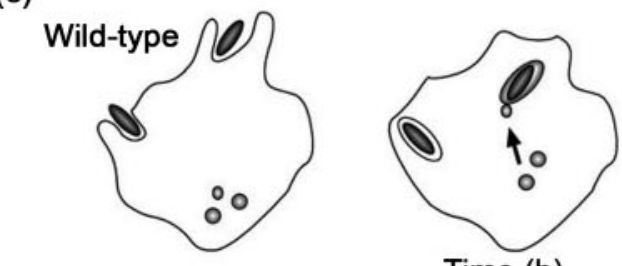

Time (h)
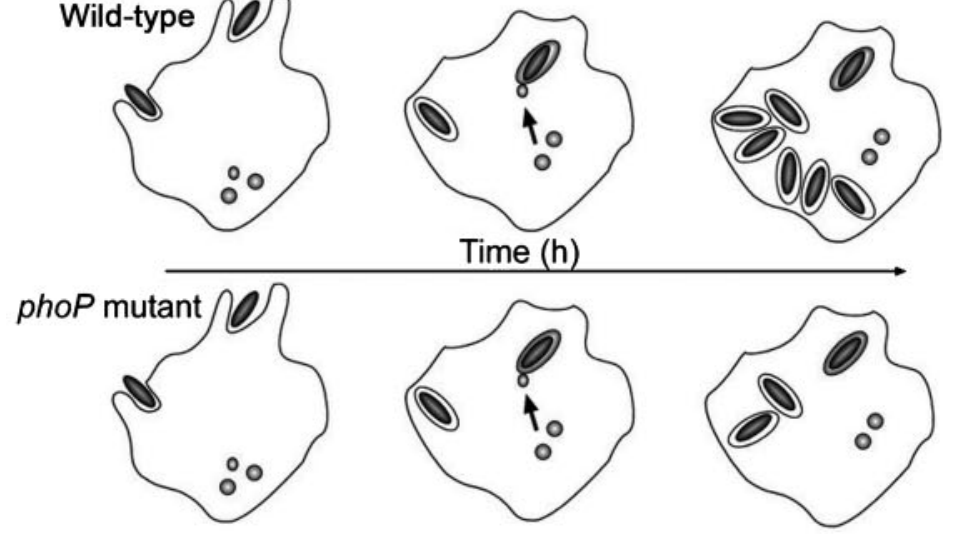

Salmonella ○ Lysosome
Fig. 5. Kinetics of SCV-lysosome interactions in RAW264.7 macrophages. (a) Mean percentage of bacteria per cell co-localized with TROva in RAW264.7 macrophages infected with wild-type, phoP mutant or heat-killed $S$. Typhimurium at the times indicated. Data shown are the means of at least three independent experiments (error bars, SD), and asterisks indicate a significant difference from the wild-type at the corresponding time point, where $P<0.05$. (b) Mean total number of bacteria per cell and mean number of TROva $^{+}$bacteria per cell in RAW264.7 macrophages infected with wild-type, phoP mutant or heat-killed $S$. Typhimurium. Data shown are the mean of at least three independent experiments (error bars, SD), and asterisks indicate a significant difference between wild-type and pho $P$ mutant bacteria, while a cross indicates a significant difference between wild-type and heat-killed bacteria, where $P<0.05$. (c) Illustration of how differences in growth rates between wild-type and mutant bacterial strains affect the interpretation of the avoidance of phagolysosomal fusion on a proportional basis. In each case only one phagosome fuses with lysosomes, but the overall growth defect of the mutant results in a higher proportion of phagosomes that have undergone fusion. 
adapt to this environment and efficiently express other factors required for survival and replication within the phagosomal environment. Resistance to AMPs and resistance to acidic $\mathrm{pH}$ are therefore likely to be important responses enabling PhoP/Q-dependent replication of Salmonella in macrophages and virulence in its hosts.

\section{ACKNOWLEDGEMENTS}

This work was supported by grants from the MRC and Wellcome Trust (UK) to D.W.H., and a BBSRC studentship to J.A.T. We thank the Salmonella research group for their critical appraisal of this manuscript.

\section{REFERENCES}

Bearson, B. L., Wilson, L. \& Foster, J. W. (1998). A low pH-inducible, PhoPQ-dependent acid tolerance response protects Salmonella typhimurium against inorganic acid stress. J Bacteriol 180, 2409-2417.

Becken, U., Jeschke, A., Veltman, K. \& Haas, A. (2010). Cell-free fusion of bacteria-containing phagosomes with endocytic compartments. Proc Natl Acad Sci U S A 107, 20726-20731.

Beuzón, C. R., Banks, G., Deiwick, J., Hensel, M. \& Holden, D. W. (1999). $\mathrm{pH}$-dependent secretion of SseB, a product of the SPI-2 type III secretion system of Salmonella typhimurium. Mol Microbiol 33, 806-816.

Beuzón, C. R., Méresse, S., Unsworth, K. E., Ruíz-Albert, J., Garvis, S., Waterman, S. R., Ryder, T. A., Boucrot, E. \& Holden, D. W. (2000). Salmonella maintains the integrity of its intracellular vacuole through the action of SifA. EMBO J 19, 3235-3249.

Beuzón, C. R., Salcedo, S. P. \& Holden, D. W. (2002). Growth and killing of a Salmonella enterica serovar Typhimurium sifA mutant strain in the cytosol of different host cell lines. Microbiology 148, 2705-2715.

Blanc-Potard, A. B. \& Groisman, E. A. (1997). The Salmonella selC locus contains a pathogenicity island mediating intramacrophage survival. EMBO J 16, 5376-5385.

Brodsky, I. E., Ernst, R. K., Miller, S. I. \& Falkow, S. (2002). mig-14 is a Salmonella gene that plays a role in bacterial resistance to antimicrobial peptides. J Bacteriol 184, 3203-3213.

Buchmeier, N. A. \& Heffron, F. (1991). Inhibition of macrophage phagosome-lysosome fusion by Salmonella typhimurium. Infect Immun 59, 2232-2238.

Craig, M. \& Slauch, J. M. (2009). Phagocytic superoxide specifically damages an extracytoplasmic target to inhibit or kill Salmonella. PLoS ONE 4, e4975.

Datsenko, K. A. \& Wanner, B. L. (2000). One-step inactivation of chromosomal genes in Escherichia coli K-12 using PCR products. Proc Natl Acad Sci U S A 97, 6640-6645.

De Groote, M. A., Ochsner, U. A., Shiloh, M. U., Nathan, C., McCord, J. M., Dinauer, M. C., Libby, S. J., Vazquez-Torres, A., Xu, Y. \& Fang, F. C. (1997). Periplasmic superoxide dismutase protects Salmonella from products of phagocyte NADPH-oxidase and nitric oxide synthase. Proc Natl Acad Sci U S A 94, 13997-14001.

Detweiler, C. S., Monack, D. M., Brodsky, I. E., Mathew, H. \& Falkow, S. (2003). virK, som $A$ and $r c s C$ are important for systemic Salmonella enterica serovar Typhimurium infection and cationic peptide resistance. Mol Microbiol 48, 385-400.

Drecktrah, D., Knodler, L. A., Ireland, R. \& Steele-Mortimer, O. (2006). The mechanism of Salmonella entry determines the vacuolar environment and intracellular gene expression. Traffic 7, 39-51.
Garin, J., Diez, R., Kieffer, S., Dermine, J. F., Duclos, S., Gagnon, E., Sadoul, R., Rondeau, C. \& Desjardins, M. (2001). The phagosome proteome: insight into phagosome functions. J Cell Biol 152, 165-180.

Garvis, S. G., Beuzón, C. R. \& Holden, D. W. (2001). A role for the PhoP/Q regulon in inhibition of fusion between lysosomes and Salmonella-containing vacuoles in macrophages. Cell Microbiol 3, 731-744.

Ghosh, P., Griffith, J., Geuze, H. J. \& Kornfeld, S. (2003). Mammalian GGAs act together to sort mannose 6-phosphate receptors. J Cell Biol 163, 755-766.

Groisman, E. A., Chiao, E., Lipps, C. J. \& Heffron, F. (1989). Salmonella typhimurium phoP virulence gene is a transcriptional regulator. Proc Natl Acad Sci U S A 86, 7077-7081.

Günzel, D., Kucharski, L. M., Kehres, D. G., Romero, M. F. \& Maguire, M. E. (2006). The MgtC virulence factor of Salmonella enterica serovar Typhimurium activates $\mathrm{Na}^{+}, \mathrm{K}^{+}$-ATPase. J Bacteriol 188, 5586-5594.

Guo, L., Lim, K. B., Poduje, C. M., Daniel, M., Gunn, J. S., Hackett, M. \& Miller, S. I. (1998). Lipid A acylation and bacterial resistance against vertebrate antimicrobial peptides. Cell 95, 189-198.

Hashim, S., Mukherjee, K., Raje, M., Basu, S. K. \& Mukhopadhyay, A. (2000). Live Salmonella modulate expression of Rab proteins to persist in a specialized compartment and escape transport to lysosomes. J Biol Chem 275, 16281-16288.

Hébrard, M., Viala, J. P., Méresse, S., Barras, F. \& Aussel, L. (2009). Redundant hydrogen peroxide scavengers contribute to Salmonella virulence and oxidative stress resistance. J Bacteriol 191, 4605-4614.

Helaine, S., Thompson, J. A., Watson, K. G., Liu, M., Boyle, C. \& Holden, D. W. (2010). Dynamics of intracellular bacterial replication at the single cell level. Proc Natl Acad Sci U S A 107, 3746-3751.

Hensel, M., Shea, J. E., Gleeson, C., Jones, M. D., Dalton, E. \& Holden, D. W. (1995). Simultaneous identification of bacterial virulence genes by negative selection. Science 269, 400-403.

Hiemstra, P. S., Eisenhauer, P. B., Harwig, S. S., van den Barselaar, M. T., van Furth, R. \& Lehrer, R. I. (1993). Antimicrobial proteins of murine macrophages. Infect Immun 61, 3038-3046.

Hiemstra, P. S., van den Barselaar, M. T., Roest, M., Nibbering, P. H. \& van Furth, R. (1999). Ubiquicidin, a novel murine microbicidal protein present in the cytosolic fraction of macrophages. J Leukoc Biol 66, 423-428.

Hmiel, S. P., Snavely, M. D., Florer, J. B., Maguire, M. E. \& Miller, C. G. (1989). Magnesium transport in Salmonella typhimurium: genetic characterization and cloning of three magnesium transport loci. J Bacteriol 171, 4742-4751.

Jahreiss, L., Menzies, F. M. \& Rubinsztein, D. C. (2008). The itinerary of autophagosomes: from peripheral formation to kiss-and-run fusion with lysosomes. Traffic 9, 574-587.

Loessner, H., Endmann, A., Leschner, S., Westphal, K., Rohde, M., Miloud, T., Hämmerling, G., Neuhaus, K. \& Weiss, S. (2007). Remote control of tumour-targeted Salmonella enterica serovar Typhimurium by the use of L-arabinose as inducer of bacterial gene expression in vivo. Cell Microbiol 9, 1529-1537.

Mastroeni, P., Vazquez-Torres, A., Fang, F. C., Xu, Y., Khan, S., Hormaeche, C. E. \& Dougan, G. (2000). Antimicrobial actions of the $\mathrm{NADPH}$ phagocyte oxidase and inducible nitric oxide synthase in experimental salmonellosis. II. Effects on microbial proliferation and host survival in vivo. J Exp Med 192, 237-248.

Miller, S. I., Kukral, A. M. \& Mekalanos, J. J. (1989). A two-component regulatory system ( $p h o P$ phoQ) controls Salmonella typhimurium virulence. Proc Natl Acad Sci U S A 86, 5054-5058.

Mills, P. C., Rowley, G., Spiro, S., Hinton, J. C. \& Richardson, D. J. (2008). A combination of cytochrome $c$ nitrite reductase (NrfA) and 
flavorubredoxin (NorV) protects Salmonella enterica serovar Typhimurium against killing by $\mathrm{NO}$ in anoxic environments. Microbiology 154, 1218-1228.

Moncrief, M. B. \& Maguire, M. E. (1998). Magnesium and the role of MgtC in growth of Salmonella typhimurium. Infect Immun 66, 38023809.

Mullock, B. M., Bright, N. A., Fearon, C. W., Gray, S. R. \& Luzio, J. P. (1998). Fusion of lysosomes with late endosomes produces a hybrid organelle of intermediate density and is NSF dependent. J Cell Biol 140, 591-601.

Mullock, B. M., Smith, C. W., Ihrke, G., Bright, N. A., Lindsay, M., Parkinson, E. J., Brooks, D. A., Parton, R. G., James, D. E. \& other authors (2000). Syntaxin 7 is localized to late endosome compartments, associates with Vamp 8, and is required for late endosomelysosome fusion. Mol Biol Cell 11, 3137-3153.

Ochman, H. \& Groisman, E. A. (1996). Distribution of pathogenicity islands in Salmonella spp. Infect Immun 64, 5410-5412.

Pryor, P. R., Mullock, B. M., Bright, N. A., Gray, S. R. \& Luzio, J. P. (2000). The role of intraorganellar $\mathrm{Ca}^{2+}$ in late endosome-lysosome heterotypic fusion and in the reformation of lysosomes from hybrid organelles. J Cell Biol 149, 1053-1062.

Rang, C., Alix, E., Felix, C., Heitz, A., Tasse, L. \& Blanc-Potard, A. B. (2007). Dual role of the MgtC virulence factor in host and non-host environments. Mol Microbiol 63, 605-622.

Rathman, M., Sjaastad, M. D. \& Falkow, S. (1996). Acidification of phagosomes containing Salmonella typhimurium in murine macrophages. Infect Immun 64, 2765-2773.

Rathman, M., Barker, L. P. \& Falkow, S. (1997). The unique trafficking pattern of Salmonella typhimurium-containing phagosomes in murine macrophages is independent of the mechanism of bacterial entry. Infect Immun 65, 1475-1485.

Rosenberger, C. M., Gallo, R. L. \& Finlay, B. B. (2004). Interplay between antibacterial effectors: a macrophage antimicrobial peptide impairs intracellular Salmonella replication. Proc Natl Acad Sci U S A 101, 2422-2427.
Sambrook, J. \& Russell, D. W. (2001). Molecular Cloning: a Laboratory Manual. Cold Spring Harbor, NY: Cold Spring Harbor Laboratory.

Schwan, W. R., Huang, X. Z., Hu, L. \& Kopecko, D. J. (2000). Differential bacterial survival, replication, and apoptosis-inducing ability of Salmonella serovars within human and murine macrophages. Infect Immun 68, 1005-1013.

Shea, J. E., Beuzon, C. R., Gleeson, C., Mundy, R. \& Holden, D. W. (1999). Influence of the Salmonella typhimurium pathogenicity island 2 type III secretion system on bacterial growth in the mouse. Infect Immun 67, 213-219.

Soncini, F. C., García Véscovi, E., Solomon, F. \& Groisman, E. A. (1996). Molecular basis of the magnesium deprivation response in Salmonella typhimurium: identification of PhoP-regulated genes. J Bacteriol 178, 5092-5099.

Sörensen, M., Lippuner, C., Kaiser, T., Misslitz, A., Aebischer, T. \& Bumann, D. (2003). Rapidly maturing red fluorescent protein variants with strongly enhanced brightness in bacteria. FEBS Lett 552, 110-114.

Testerman, T. L., Vazquez-Torres, A., Xu, Y., Jones-Carson, J., Libby, S. J. \& Fang, F. C. (2002). The alternative sigma factor $\sigma^{\mathrm{E}}$ controls antioxidant defences required for Salmonella virulence and stationary-phase survival. Mol Microbiol 43, 771-782.

Uchiya, K., Barbieri, M. A., Funato, K., Shah, A. H., Stahl, P. D. \& Groisman, E. A. (1999). A Salmonella virulence protein that inhibits cellular trafficking. EMBO J 18, 3924-3933.

Vazquez-Torres, A., Jones-Carson, J., Mastroeni, P., Ischiropoulos, H. \& Fang, F. C. (2000a). Antimicrobial actions of the NADPH phagocyte oxidase and inducible nitric oxide synthase in experimental salmonellosis. I. Effects on microbial killing by activated peritoneal macrophages in vitro. J Exp Med 192, 227-236.

Vazquez-Torres, A., Xu, Y., Jones-Carson, J., Holden, D. W., Lucia, S. M., Dinauer, M. C., Mastroeni, P. \& Fang, F. C. (2000b). Salmonella pathogenicity island 2-dependent evasion of the phagocyte NADPH oxidase. Science 287, 1655-1658.

Edited by: D. L. Gally 